\title{
Impact of nano-sized ceria particles upon the cyclization kinetics of poly(amic acid) films
}

\author{
I. V. Gofman ${ }^{1}$, E. N. Vlasova ${ }^{1}$, A. L. Nikolaeva ${ }^{1}$, A. V. Yakimansky ${ }^{1,2}$, \\ O. S. Ivanova ${ }^{3}$, A. E. Baranchikov ${ }^{3}$, V. K. Ivanov ${ }^{3}$ \\ ${ }^{1}$ Institute of Macromolecular Compounds, Russian Academy of Sciences, \\ 199004, Bolshoi prospect 31, Saint Petersburg, Russia \\ ${ }^{2}$ Saint Petersburg State University, Institute of Chemistry, \\ 198504, Universitetskii prospect 26, Peterhof, Saint Petersburg, Russia \\ ${ }^{3}$ Kurnakov Institute of General and Inorganic Chemistry, Russian Academy of Sciences, \\ 119991, Leninsky prospect 31, Moscow, Russia \\ gofman@imc.macro.ru, ev1021960@gmail.com, alexandra.1.nikolaeva@gmail.com, yakimansky@yahoo.com, \\ runetta05@mail.ru, a.baranchikov@yandex.ru,van@igic.ras.ru
}

PACS 81.07.-b, 62.25.-g

DOI 10.17586/2220-8054-2019-10-4-475-479

The experimental study was conducted of the impact of ceria nanoparticles introduced into poly(amic acid), the prepolymer of thermally stable aromatic polyimide, upon the kinetics of the thermal cyclization of the film of this prepolymer, the reaction of its transformation to the final polyimide film in the course of its thermal treatment. The nano-filler was shown to accelerate the initial stage of cyclization, occurring at temperatures ranging up to $100-120^{\circ} \mathrm{C}$. A possible way of practical application of this effect was discussed.

Keywords: polymer-inorganic nanocomposites, polyimide, poly(amic acid), ceria, cyclization, mechanical properties.

Received: 5 August 2019

\section{Introduction}

Among different groups of polymer-inorganic nanocomposite materials that are the subject of extensive studies in last decades, some interesting properties are inherent to the composites obtained by introduction of nano-sized particles of ceria $[1,2]$ in matrices of thermally stable aromatic polyimides (PIs). It was shown in the previous investigations of these materials that the ceria nanoparticles of quasi-spherical shape do not produce any substantial positive impact on both their mechanical properties and glass transition temperature, but they provoke a sizable variation of the thermal stability of matrix PI [3].

In the communication presented, the results of the following stage of the investigations of PI-ceria nanocomposites are presented, namely the impact of this nano-filler upon the kinetics of synthesis of PI-based film materials is analyzed. The results are examined from a comparative study of the kinetics of the final stage of PI synthesis, the cyclization of prepolymer, a poly(amic acid) (PAA) [4] on one hand, and of the nanocomposite based on this PAA, in which ceria nanoparticles were introduced as an active nano-sized filler, on the other.

\section{Materials and methods}

A prepolymer used in our work was the PAA of the following structure:

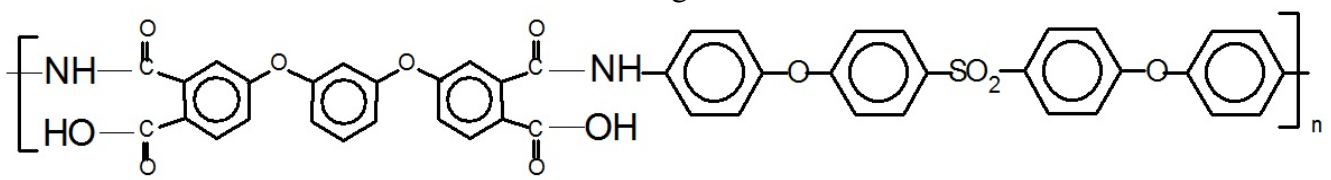

The PAA solution in N-methylpyrrolidone was synthesized in Institute of Macromolecular Compounds, Russian Academy of Sciences. The synthetic protocol was described previously [4].

The thermal treatment of the films of this PAA up to $250-300{ }^{\circ} \mathrm{C}$ results in the curing (cyclization) reaction in which the thermally stable aromatic polyimide R-BAPS is formed:

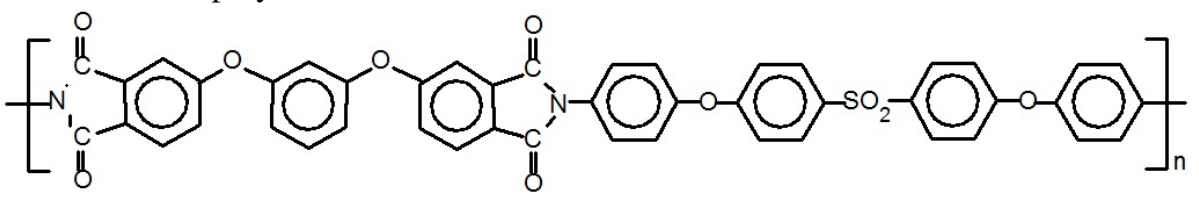

Quasi-spherical ceria nanoparticles with a diameter of $\sim 3-4 \mathrm{~nm}$, which were used to prepare the nanocomposite, were synthesised at the Kurnakov Institute of General and Inorganic Chemistry, Russian Academy of Sciences. The 
protocol for their synthesis is described elsewhere [5]. Briefly, cerium(III) nitrate $(0.08 \mathrm{~mol} / \mathrm{L})$ in a water/isopropanol $(1: 1)$ mixture $(180 \mathrm{~mL})$ was added to aqueous ammonia $(3 \mathrm{~mol} / \mathrm{L}, 900 \mathrm{~mL})$. The mixture was vigorously stirred for 3 h. Yellow precipitate was washed with distilled water and dried overnight at $60{ }^{\circ} \mathrm{C}$.

To form the nanocomposite film samples, a pre-calculated amount of ceria nanoparticles was sonicated in $\mathrm{N}$ methylpyrrolidone, and then a pre-calculated amount of PAA solution was introduced to the obtained nanoparticle dispersion. The mixture was then subjected to a long-term $(24 \mathrm{~h})$ homogenization by a mechanical stirrer (1000 rpm). The layers of homogenized PAA-based nanocomposite solution or of control unfilled PAA solution were cast onto glass plates with the subsequent drying at $80{ }^{\circ} \mathrm{C}$ for $24 \mathrm{~h}$ in a vacuum to completely remove the solvent. The ceria concentration in PAA-ceria nanocomposite as high as $3 \mathrm{wt} \%$ was chosen for this investigation.

The PAA or PAA- $\mathrm{CeO}_{2}$ nanocomposite films obtained by this way were subjected to a stepwise thermal treatment at the temperatures of $80,90,100,120,140,160,200$ and $250{ }^{\circ} \mathrm{C}$. Each step lasted $30 \mathrm{~min}$. After each step of treatment the IR spectra of films were recorded and the mechanical tests were carried out.

Fourier-transform IR spectra of the films were recorded by using the "Vertex70" spectrometer (Bruker) equipped by a "Pike" micro-unit of singly disturbed total internal reflection with the ZnSe working element. Frequency resolution in these tests was of $4 \mathrm{~cm}^{-1}$, scans number -30 . While registering the spectra, the correction possibility was envisaged to take into account the penetration depth dependence upon the wavelength, but in our experiments the "Pike" micro-fixture used insures the constant sample's pressing value and, hence, the constant penetration depth of IR irradiation into the sample. This makes it possible to carry out direct comparison of the obtained spectra.

The variation of the degree of cyclization of both PAA and $\mathrm{PAA}-\mathrm{CeO}_{2}$ composite films during their heating with the increase of temperature was quantitatively estimated by the increase in the concentration of imide cycles (the rise of the intensity of spectral band at $1778 \mathrm{~cm}^{-1}$, which corresponds to the symmetric valent vibrations of $\mathrm{C}=\mathrm{O}$ bond) and by the decrease of the concentration of amic acid groups (the fall of the intensity of spectral band $1540 \mathrm{~cm}^{-1}$ corresponding to $\mathrm{CNH}$ vibrations). Optical density values for the above spectral bands were then normalized to that of the intrinsic standard: the $1015 \mathrm{~cm}^{-1}$ band (the vibrations of aromatic ring) that does not vary during the cyclization process [4].

Thermogravimetric tests of the materials to control the residual amount of the solvent in dried films were conducted by a DTG-60 setup (Shimadzu, Japan). Film samples (approximately $5 \mathrm{mg}$ ) were heated to $350{ }^{\circ} \mathrm{C}$ at a rate of $5{ }^{\circ} \mathrm{C} / \mathrm{min}$. The experiments were carried out in an air atmosphere.

The mechanical tests of the films under investigation were carried out in the uniaxial extension mode, at room temperature, using an AG-100kNX Plus universal mechanical test system (Shimadzu, Japan). Strip-like samples with the dimensions $2 \times 30 \mathrm{~mm}$ were stretched at a rate of $5 \mathrm{~mm} / \mathrm{min}$, according to ASTM D638 requirements. The Young's modulus values $E$ were determined in these tests.

\section{Results}

IR spectra of PAA film and PAA-ceria nanocomposite one at the early stage of cyclization: after the thermal treatment at $90{ }^{\circ} \mathrm{C}$, are presented in Fig. 1 (Fig. 1a). In the same figure (Fig. 1b), the spectra are presented for the completely cured films - after treatment at $250{ }^{\circ} \mathrm{C}$.

The spectra obtained testify to the acceleration of cyclization process in the PAA film filled with ceria, as compared to that in unfilled PAA film: the concentration of imide rings in the former increases substantially faster along with the increase in temperature than that in the unfilled PAA film. For example, after the thermal treatment of PAA-ceria nanocomposite film at $90{ }^{\circ} \mathrm{C}$ the degree of cyclization of PAA-ceria nanocomposite film is three times more than that of the PAA film. The same conclusion was drawn by comparing the speeds of the decrease of the concentrations of amic acid units in the samples studied (Fig. 1a).

But the increased intensity of the process is inherent to the nanocomposite film only at the initial stage of the PAA thermal curing process, namely at temperatures up to $100-120{ }^{\circ} \mathrm{C}$. During the further heating of films of two types, the degrees of cyclization became similar, and up to $250{ }^{\circ} \mathrm{C}$, the PAA conversion to PI completely finishes in both materials (within the accuracy of the conversion degree determination by spectral method).

To confirm the results of IR tests by some other independent method, both the PAA films and PAA-based nanocomposite ones were subjected to the mechanical test after thermal treatment at different temperatures. The value of interest in these tests was the Young's modulus of the film materials. It is known that a system of H-bonds involving the $\mathrm{CONH}$ and $\mathrm{COOH}$ groups of the amic acid units is formed in the uncured PAA films [4]. But during the cyclization process, these bonds are broken. As a result, there are no H-bonds in the products of cyclization process, the PI films [4]. Under this reason, the cyclization process in PAA is accompanied by some modest decrease of the Young's modulus of the film. This decrease can hardly be fixed as usual at the expense of the incomplete removal of the solvent from the starting polymer volume during the PAA film drying process under standard conditions. This solvent (as a rule it is $\mathrm{N}, \mathrm{N}$ - dimethylformamide, N,N- dimethylacetamide, or N-methylpyrrolidone) acts as a plasticizer 

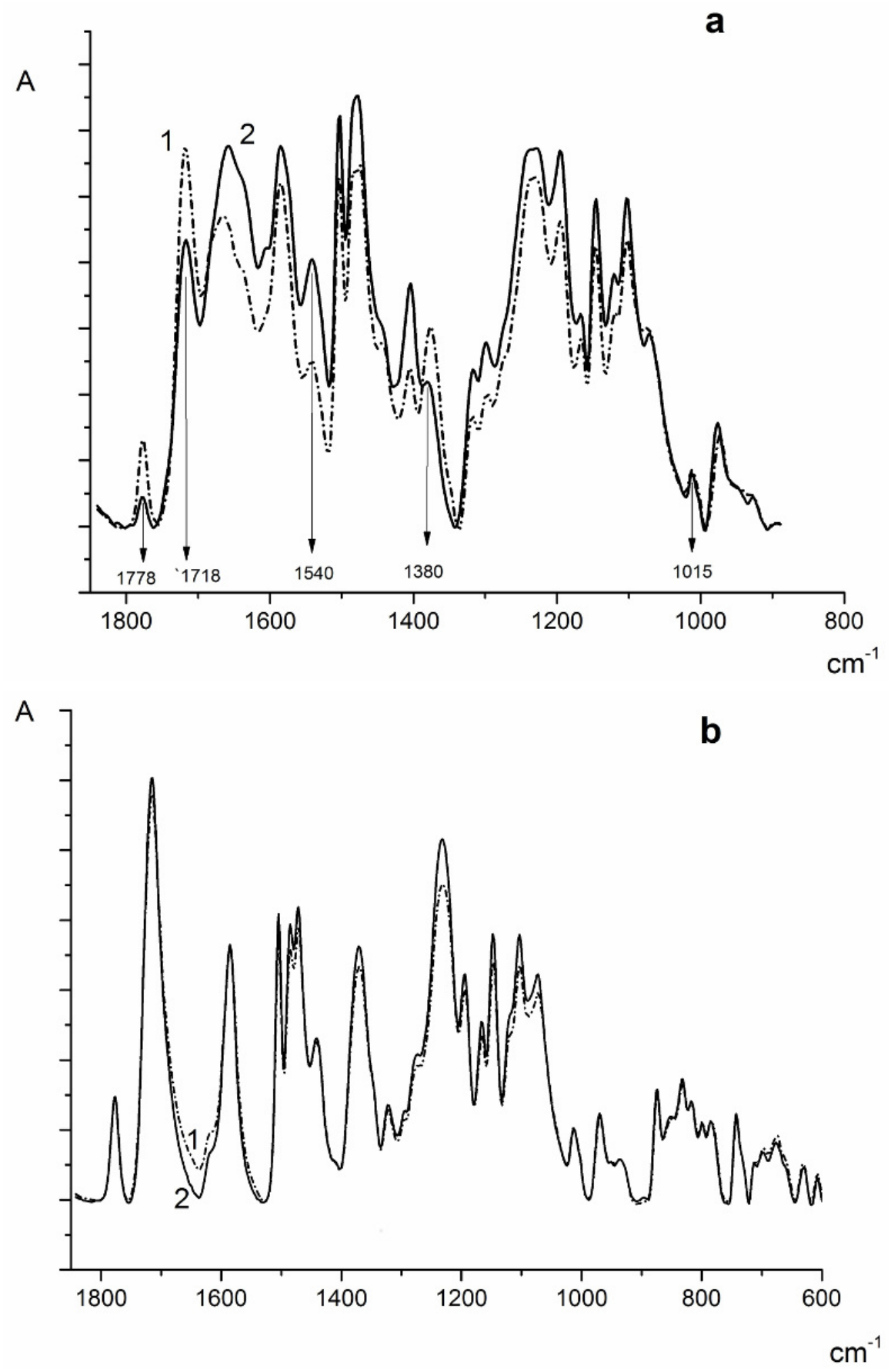

FIG. 1. IR spectra of (a) PAA and PAA- $\mathrm{CeO}_{2}$ films after heat treatment at $90{ }^{\circ} \mathrm{C}$; (b) PI and PI$\mathrm{CeO}_{2}$ films after thermal treatment at $250{ }^{\circ} \mathrm{C}$. (1) $-\mathrm{PAA}-\mathrm{CeO}_{2}$ or $\mathrm{PI}-\mathrm{CeO}_{2}$ nanocomposite films, (2) - PAA or PI films 
in the film, which causes a decrease in the initial value of Young's modulus. During the subsequent thermally stimulated PAA cyclization process, the vaporization of this residual solvent occurs causing an increase in the modulus. As a result of the interplay of two processes above - the solvent removal and the destruction of H-bonds during heat treatment of the film - the resulting Young's modulus value of cured film does not decrease and moreover, can slightly increase as compared to that of the PAA film.

To exclude the impact of the deplastification process upon the variation of the Young's modulus during the thermal cyclization, a special pretreatment of both PAA and PAA-based nanocomposite films was carried out; they were subjected to prolonged drying under vacuum (the drying protocol is described above). The completion of the drying process was controlled by TGA tests. The weight losses registered by this method during the heating of pretreated films in the temperature range $80-300{ }^{\circ} \mathrm{C}$ were found to be similar to the calculated amount of water, the side product of PAA cyclization reaction. Hence, pretreatment insures the efficient solvent removal from the films and eliminates the impact of deplastification effects upon the results of our tests.

The curves of Young's modulus values of PAA and PAA-ceria nanocomposite films vs. the temperature in the course of the stepwise thermal treatment of the films are presented in Fig. 2.

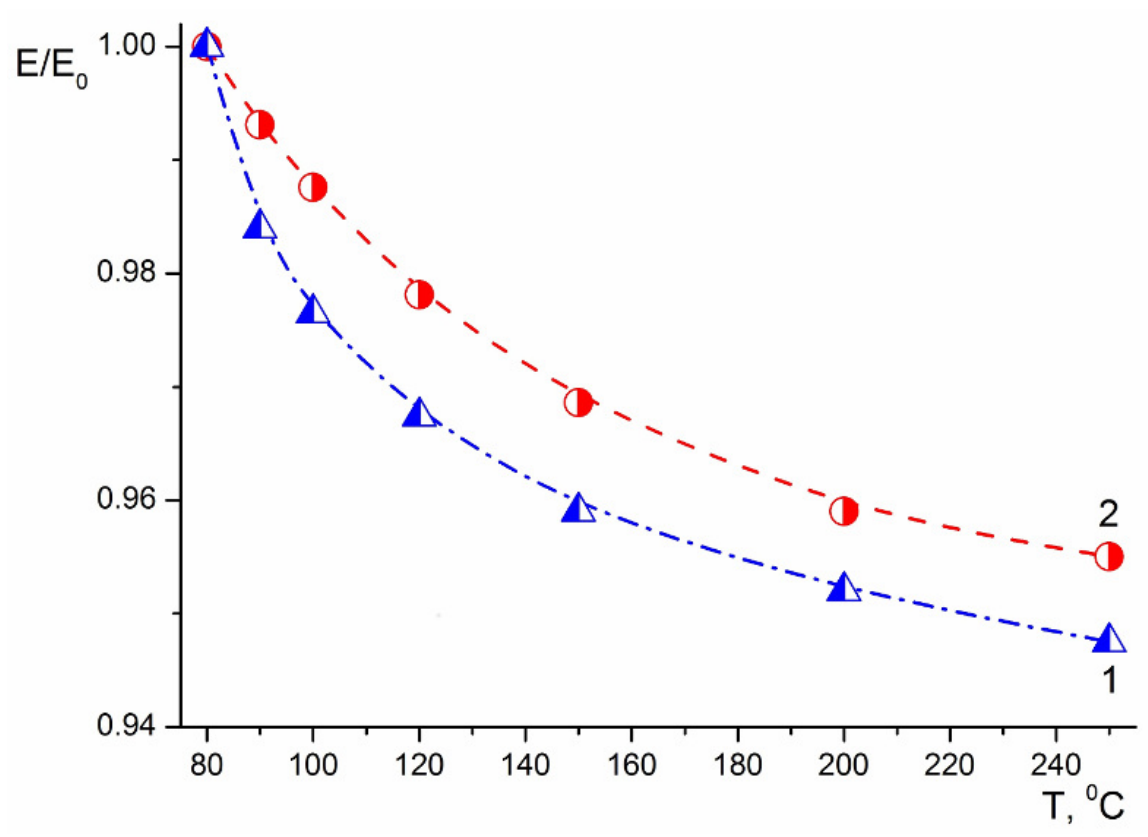

FIG. 2. Young's modulus values of PAA-based composite and PAA films vs. temperature of thermal treatment during the conversion of prepolymer to R-BAPB-based nanocomposite containing $3 \mathrm{wt}$ \% of $\mathrm{CeO}_{2}$ (1) or to PI R-BAPB (2); $\mathrm{E}_{0}$ denotes the initial $\mathrm{E}$ value of the prepolymer (PAA or PAAceria) film pretreated at $80{ }^{\circ} \mathrm{C}$

The results obtained clearly demonstrate the decrease in the Young's modulus values of both PAA and PAA-based nanocomposite films during thermal conversion to PI and PI-based composite ones. At the same time curves in Fig. 2 testify the increased speed of the E depression of nanocomposite film in the initial stage of the process as compared to that of PAA film.

In this way, the data presented evidence the catalytic action of ceria nanoparticles exfoliated in PAA volume on the process of thermally stimulated cyclization of film of this PAA. Indeed, this catalytic impact was registered only at the initial stage of the conversion process: at temperatures ranging up to $100-120^{\circ} \mathrm{C}$. During the further heating of both films the equal degrees of conversion were registered in both films.

At first glance, the effect above is of no practical interest, because it is realized only at the first, initial stage of conversion of PAA to PI. But as a matter of fact, this phenomenon lets us to decrease the severity of the requirements for the initial molecular mass of PAA, which insures the successful fabrication of PI films. Indeed, during the thermal treatment of PAA films, some side reaction takes place along with the cyclization. This is a reaction of thermally stimulated destruction, cleavage of PAA macrochains [4], i.e., the successive decrease of PAA molecular mass. This is a reversible process that occurs at temperatures of $100-150{ }^{\circ} \mathrm{C}$. During the further heating of film, the destruction process gives way to the opposite one, the re-synthesis with the successive increase in the molecular mass of the polymer. Under the optimal conditions of the cyclization process, the full re-synthesis takes place in the temperature 
range up to $180-200{ }^{\circ} \mathrm{C}$, resulting in the full restoration of molecular mass up to its initial value [4]. But in the temperature interval $100-150{ }^{\circ} \mathrm{C}$ the several fold (up to one order) decrease of molecular mass as compared to its initial value takes place. If this initial value is not very high, the destruction process can provoke the damage of the film in the aforementioned range of temperature at the expense of the local stresses caused by the removal of the solvent.

But if some amount of cured units, of imide rings will be formed in the film up to the development of the destruction process, as in the PAA-ceria nanocomposite film, this can insure the depression of the destruction intensity and hence the possibility increases for successful completion of thermally stimulated cyclization and re-synthesis without the film's damage at the intermediate stages of curing process.

\section{Acknowledgment}

The authors acknowledge the help of L.A. Myagkova from the Institute of Macromolecular Compounds, RAS, who synthesised the prepolymer of the matrix polymer R-BAPS.

The work was supported by the Russian Science Foundation (project No. 18-13-00305).

\section{References}

[1] Ivanov V.K., Polezhaeva O.S., et al. Synthesis and thermal stability of nanocrystalline ceria sols stabilized by citric and polyacrylic acids. Russian Journal of Inorganic Chemistry, 2010, 55(3), P. 328-332.

[2] Mullins D.R. The surface chemistry of cerium oxide. Surface Science Reports, 2015, 70(1), P. $42-85$.

[3] Gofman I., Nikolaeva A., et al. Unexpected selective enhancement of the thermal stability of aromatic polyimide materials by cerium dioxide nanoparticles. Polymers for advanced technologies, 2019, 30(6), P. 1518-1524.

[4] Bessonov M.I., Koton M.M., Kudryavtsev V.V., Laius L.A. Polyimides - thermally stable polymers. Plenum Publishing Corp., New York, $1987,327 \mathrm{p}$

[5] Ivanov V.K., Kopitsa G.P., et al. Hydrothermal growth of ceria nanoparticles. Russian Journal of Inorganic Chemistry, 2009, 54(12), P. 18571861. 